INVESTIGACIÓN

Recibido: 09/10/2016 --- Aceptado: 19/01/2017 --- Publicado: 15/06/2017

\title{
PERSPECTIVAS DE LOS ESTUDIANTES SOBRE EL USO DE REDES SOCIALES EN EL PROCESO EDUCATIVO SUPERIOR
}

\section{Perspectives of the students about the use of networks in the higher educational process}

Ricardo Adán Salas Rueda ${ }^{\mathbf{1}}$ Universidad La Salle. México.

ricardo.salas@ulsa.mx

José Luis Lugo García: Universidad La Salle. México.

joseluis.lugo@ulsa.mx

Héctor Fernando Ruiz Silva: Universidad La Salle. México.

fernandoruiz.it@gmail.com

\section{RESUMEN}

Las universidades en México están incorporando la tecnología durante la realización de las actividades educativas. De hecho, los docentes identifican y seleccionan las herramientas informáticas y aplicaciones de comunicación que permitan el desarrollo de las competencias en los estudiantes. En particular, esta investigación por medio del Enfoque cuantitativo analiza el impacto de utilizar la red social Facebook en el contexto educativo. La muestra está compuesta por 19 alumnos que cursan la Licenciatura en Gestión de Negocios y Tecnologías de Información en una universidad ubicada en la Ciudad de México. El instrumento de medición consta de 12 preguntas sobre la atención, la utilidad, el aprendizaje y la motivación al utilizar Facebook durante el ciclo escolar 2016-1. Por último, esta investigación afirma que las redes sociales mejoran la comunicación y facilitan la asimilación y el uso del conocimiento.

\section{PALABRAS CLAVE}

Educación Superior - Tecnología - Redes sociales - Facebook - Enseñanza Aprendizaje - Nuevas tecnologías.

\footnotetext{
${ }^{1}$ Ricardo Adán Salas Rueda: Doctor en Diseño de Nuevas Tecnologías por la Universidad Autónoma Metropolitana (UAM) en el año 2014. Ha impartido cursos en el nivel de licenciatura y posgrado en México. Candidato a investigador nacional por parte del Sistema Nacional de Investigadores (SNI). ricardo.salas@ulsa.mx
} 
Salas Rueda, R. I., Lugo García, J. L., Ruiz Silva, H. F. Perspectivas de los estudiantes sobre el uso de redes sociales en el proceso educativo superior

\begin{abstract}
Universities in Mexico are incorporating the technology for the realization of the educational activities. In fact, professors identify and select the tools and communication applications that enable the development of skills in the students. In particular, this research through the quantitative approach analyzes the impact of using the social network Facebook in the educational context. The sample consists of 19 students who are studying the career of Business Management and Information Technology at a university in Mexico City. The measuring instrument is composed of 12 questions about attention, utility, learning and motivation in using Facebook during the 2016-1 school year. Finally, this research establishes that social networks improve communication and facilitate the assimilation and use of knowledge.
\end{abstract}

\title{
KEY WORDS
}

Higher Education - Technology - Social Networking - Facebook - Teaching Learning - New technologies.

\section{PERSPECTIVAS DOS ESTUDANTES SOBRE O USO DAS REDES SOCIAIS NO PROCESSO EDUCATIVO SUPERIOR}

\section{RESUMO}

As universidades no México estão incorporando a tecnologia durante a realização das atividades educativas. De fato, os docentes identificam e selecionam as ferramentas informáticas e aplicações de comunicação que permitam o desenvolvimento das competências nos estudantes. Em particular, esta investigação por meio do Enfoque quantitativo analisa o impacto de utilizar a rede social Facebook no contexto educativo. A amostra esta composta por 19 alunos que cursam a Licenciatura em Gestão de Negócios e Tecnologias de Informação em uma universidade localizada na Cidade do México. O instrumento de medição consta de 12 perguntas sobre a atenção, a utilidade, a aprendizagem e a motivação ao utilizar Facebook durante o ciclo escolar 2016-1. Por ultimo, esta investigação afirma que as redes sociais melhoram a comunicação e facilitam a assimilação e o uso do conhecimento.

\section{PALAVRAS CHAVE}

Educação Superior - Tecnologia - Redes Sociais - Facebook - Ensinamentos Aprendizagem - Novas Tecnologias.

\section{Cómo citar este artículo}

Salas Rueda, R. I., Lugo García, J. L., Ruiz Silva, H. F. Perspectivas de los estudiantes sobre el uso de redes sociales en el proceso educativo superior [Perspectives of the students about the use of networks in the higher educational process]. Vivat academia, revista de comunicacion, 139, 53-66. Doi: https://doi.org/10.15178/va.2017.139.53-66 Recuperado de http://www.vivatacademia.net/index.php/vivat/article/view/1008 
Salas Rueda, R. I., Lugo García, J. L., Ruiz Silva, H. F. Perspectivas de los estudiantes sobre el uso de redes sociales en el proceso educativo superior

\section{INTRODUCCIÓN}

Hoy en día, las actividades y responsabilidades de los docentes y estudiantes en el salón de clases están cambiando debido a la incorporación y el uso de la tecnología durante el proceso de enseñanza-aprendizaje (González, Tornel y Jiménez, 2014; Reigeluth, 2012). De hecho, el rol de los profesores se está transformado y adaptando a las demandas y necesidades de la Sociedad del Siglo XXI provocando el surgimiento de los facilitadores (Reigeluth, 2012). Incluso, los estudiantes universitarios están participando activamente en el proceso educativo a través de las herramientas informáticas y aplicaciones de comunicación (González, Tornel y Jiménez, 2014).

Cabe mencionar que la educación a distancia ha evolucionado a través de las siguientes generaciones: la enseñanza por correspondencia, la enseñanza multimedia y la enseñanza telemática (García, 1999; Simonson, Smaldino, Albright y Zvacek, 2012). Estas transformaciones tecnológicas han propiciando que las instituciones de educación superior reconozcan la importancia de las redes sociales como mecanismos innovadores de enseñanza-aprendizaje (Valerio, Herrera, Villanueva, Herrera y Rodríguez, 2015).

Resulta valioso mencionar que "la utilización de herramientas de la red no diseñadas explícitamente para el aprendizaje tienen un gran potencial. En la medida que los profesores conozcamos las diferentes plataformas y herramientas que la red nos proporciona podemos utilizarlas con nuestros alumnos" (Martínez, 2014, p. 275). De ahí que la educación a distancia permita crear experiencias de aprendizaje por medio del uso de los sistemas de telecomunicación (Simonson, Smaldino, Albright y Zvacek, 2012).

En particular, el uso de las herramientas sociales permite compartir la información y propicia la creación de escenarios adecuados para la difusión del conocimiento (Altinay, Saner, Bahcelerli y Altinay, 2016). De acuerdo con Huang, Wu, She y Lin (2014), Facebook y MySpace son los métodos de comunicación a distancia más populares entre los usuarios de la web. El origen del uso de las redes sociales en el contexto educativo está vinculado con las herramientas Web 2.0 (Ersoy y Güneyli, 2016; Huang, Yang, Huang y Hsiao, 2010).

Asimismo Rojas (2014, p. 9) explica que "el uso de dispositivos de alta tecnología es inherente a las actividades diarias de los estudiantes universitarios. A través de los sistemas de redes sociales, en especial de Facebook, tales dispositivos se convierten en el punto de encuentro de la red de amigos y contactos directos e indirectos".

Del mismo modo, Olarte, Domínguez, Elizondo y García (2014) destacan la importancia de utilizar la red social Facebook durante el proceso de enseñanzaaprendizaje para lograr el desarrollo y la autonomía de los estudiantes. Por consiguiente, existe una necesidad de promover el uso de redes sociales en las universidades (Gallardo, Marqués y Bullen, 2015). Con este panorama en mente, esta 
Salas Rueda, R. I., Lugo García, J. L., Ruiz Silva, H. F. Perspectivas de los estudiantes sobre el uso de redes sociales en el proceso educativo superior

investigación propone analizar el impacto de utilizar la red social Facebook en una universidad ubicada en la Ciudad de México durante el proceso de enseñanzaaprendizaje relacionado con la informática.

\section{OBJETIVOS}

Esta investigación tiene como objetivo general analizar y evaluar el impacto de utilizar la red social Facebook durante el proceso de enseñanza-aprendizaje relacionado con la informática. Las variables utilizadas en este estudio son la atención, la utilidad, el aprendizaje y la motivación.

\section{METODOLOGÍA}

La metodología empleada en esta investigación se apoya en el Enfoque cuantitativo para analizar el impacto de utilizar la red social Facebook durante el proceso educativo superior. La muestra consta de 19 estudiantes que cursan la Licenciatura en Gestión de Negocios y Tecnologías de Información en una universidad ubicada en la Ciudad de México.

En este estudio, la red social Facebook es utilizada como medio para la difusión, entrega y discusión de dos actividades sobre la informática. En la primera actividad (teórica), los estudiantes emplean esta red social para compartir las respuestas de tres preguntas relacionadas con la construcción de los sistemas web. Durante la segunda actividad, los alumnos presentan el código fuente y las pantallas de salida correspondientes al desarrollo de software.

Por medio de la Estadística descriptiva se analizan y evalúan las variables sobre la atención, la utilidad, el aprendizaje y la motivación al utilizar la red social Facebook en el contexto educativo. La Tabla 1 muestra el instrumento de investigación, el cual utiliza como escala: Muy de acuerdo (1), De acuerdo (2), Ni de acuerdo ni en desacuerdo (3), Desacuerdo (4) y Muy en desacuerdo (5).

Tabla 1.

Instrumento de medición.

\begin{tabular}{|c|c|c|}
\hline No & Variable & Pregunta \\
\hline \multirow{3}{*}{1} & \multirow{3}{*}{ Atención } & Facebook captó mi atención \\
\hline & & $\begin{array}{l}\text { La organización de la información empleados en Facebook contribuyó a } \\
\text { mantener mi atención. }\end{array}$ \\
\hline & & $\begin{array}{l}\text { La variedad de los contenidos empleados en Facebook ayudó a que } \\
\text { mantuviera la atención. }\end{array}$ \\
\hline \multirow{3}{*}{2} & \multirow{3}{*}{ Utilidad } & El uso de Facebook resulta interesante. \\
\hline & & Cuando ingresé a Facebook me pareció que iba a ser fácil de utilizar. \\
\hline & & Disfruté el contenido del curso por medio de Facebook. \\
\hline \multirow{3}{*}{3} & \multirow{3}{*}{ Aprendizaje } & Mientras empleaba Facebook estaba seguro de que aprendería. \\
\hline & & $\begin{array}{l}\text { La buena organización de Facebook me permitió tener confianza de que } \\
\text { puedo aprender. }\end{array}$ \\
\hline & & Los contenidos y la estructura de Facebook son fáciles de entender. \\
\hline
\end{tabular}


Salas Rueda, R. I., Lugo García, J. L., Ruiz Silva, H. F. Perspectivas de los estudiantes sobre el uso de redes sociales en el proceso educativo superior

\begin{tabular}{|l|l|l|}
\hline \multirow{3}{*}{4} & \multirow{3}{*}{ Motivación } & Me gustaría saber más sobre el tema discutido en Facebook. \\
\cline { 3 - 3 } & Me siento satisfecho del uso de la red social Facebook. \\
\cline { 3 - 3 } & $\begin{array}{l}\text { Había contenidos en Facebook que me mostraron que este material } \\
\text { puede ser importante para otras personas. }\end{array}$ \\
\hline
\end{tabular}

Fuente: Elaboración propia.

\section{RESULTADOS Y DISCUSIÓN}

A continuación se muestran los resultados sobre la variable "Atención" para las preguntas 1, 2 y 3 del cuestionario. El Gráfico 1 muestra los resultados obtenidos para la Pregunta 1: Facebook captó mi atención. El mayor porcentaje corresponde a la categoría De acuerdo con el $47.37 \%$.

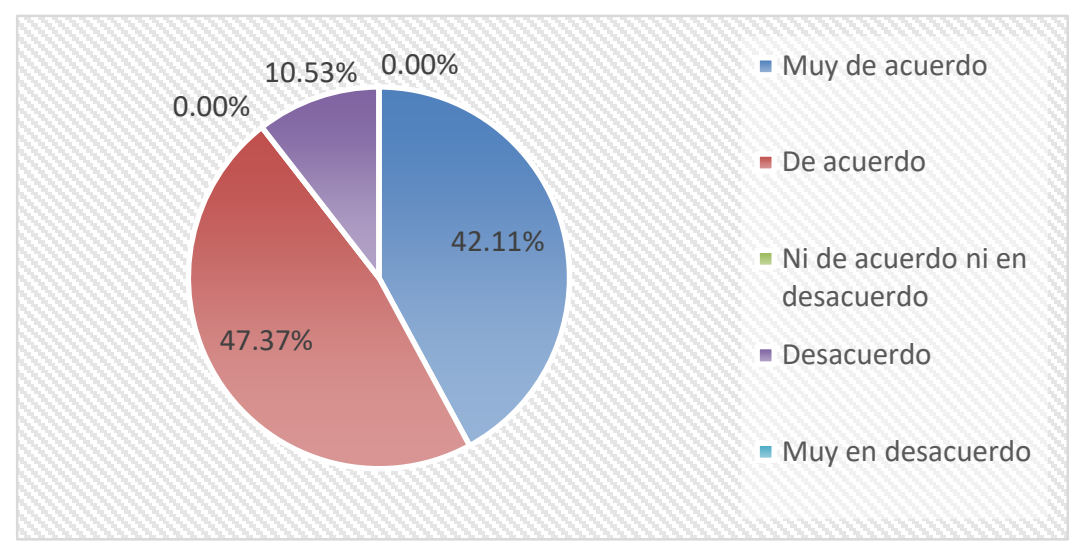

Gráfico 1. Resultados de la Pregunta 1.

Fuente: Elaboración propia.

El Gráfico 2 muestra los resultados obtenidos para la Pregunta 2: La organización de la información empleados en Facebook contribuyó a mantener mi atención. La categoría $\mathrm{Ni}$ de acuerdo ni en desacuerdo presenta la mayor preferencia con el $36.84 \%$. En segundo lugar se encuentra la categoría De acuerdo con el 31.58\%.

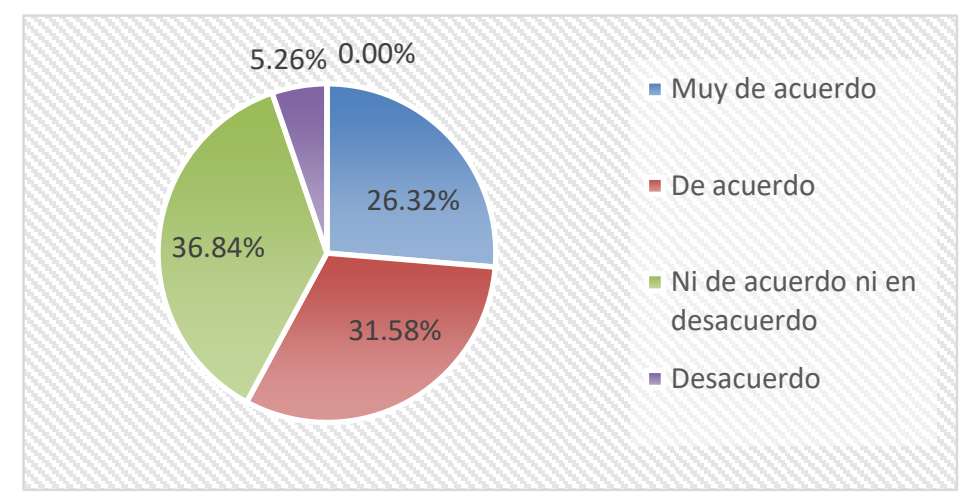

Gráfico 2. Resultados de la Pregunta 2.

Fuente: Elaboración propia. 
Salas Rueda, R. I., Lugo García, J. L., Ruiz Silva, H. F. Perspectivas de los estudiantes sobre el uso de redes sociales en el proceso educativo superior

El Gráfico 3 muestra los resultados obtenidos para la Pregunta 3: La variedad de los contenidos empleados en Facebook ayudó a que mantuviera la atención. Existe un gran porcentaje de estudiantes $(68.42 \%)$ que se ubican en la categoría De acuerdo.

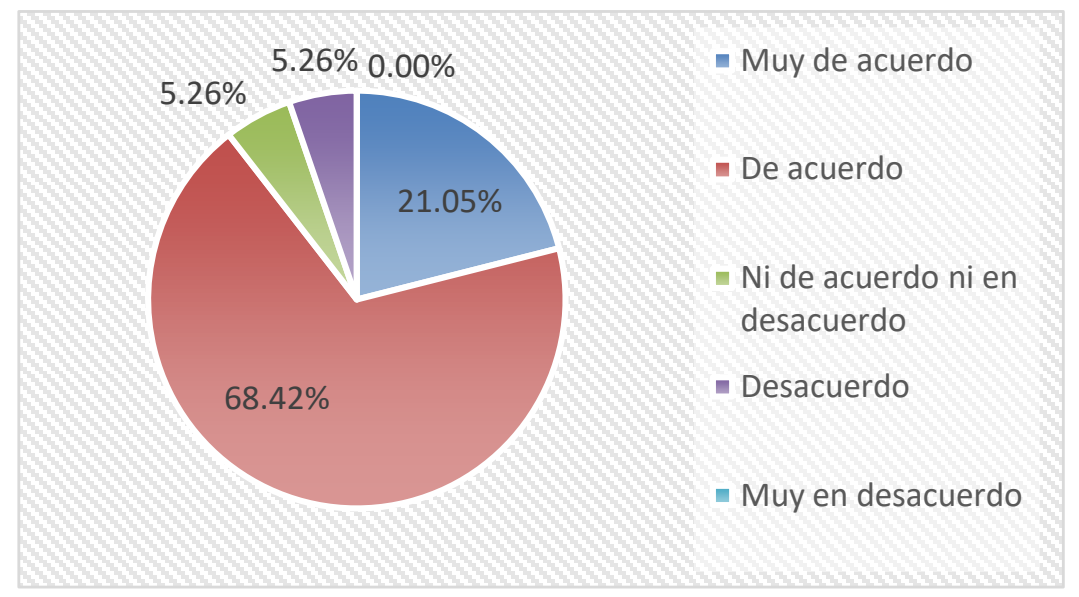

Gráfico 3. Resultados de la Pregunta 3.

Fuente: Elaboración propia.

A continuación se muestran los resultados sobre la variable "Utilidad" para las preguntas 4,5 y 6 del cuestionario.

El Gráfico 4 muestra los resultados obtenidos para la Pregunta 4: El uso de Facebook resulta interesante. Las categorías Muy de acuerdo y De acuerdo poseen el porcentaje de aceptación del $42.11 \%$.

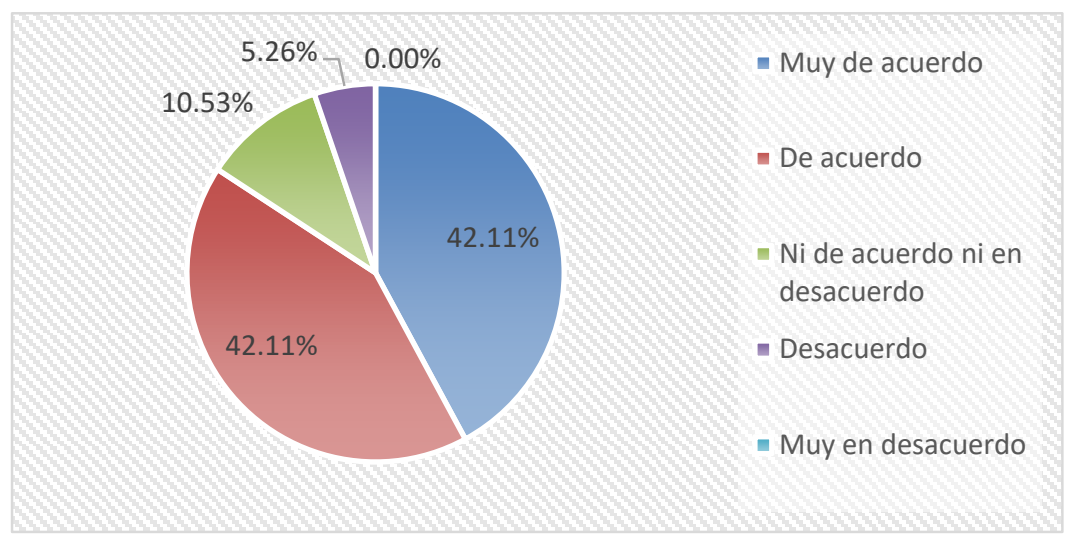

Gráfico 4. Resultados de la Pregunta 4.

Fuente: Elaboración propia.

El Gráfico 5 muestra los resultados obtenidos para la Pregunta 5: Cuando ingresé a Facebook me pareció que iba a ser fácil de utilizar. El $47.37 \%$ de los encuestados consideran que esta red social es fácil de emplear. Del mismo modo, el 31.58\% de los estudiantes se ubican en la categoría De acuerdo. 
Salas Rueda, R. I., Lugo García, J. L., Ruiz Silva, H. F. Perspectivas de los estudiantes sobre el uso de redes sociales en el proceso educativo superior

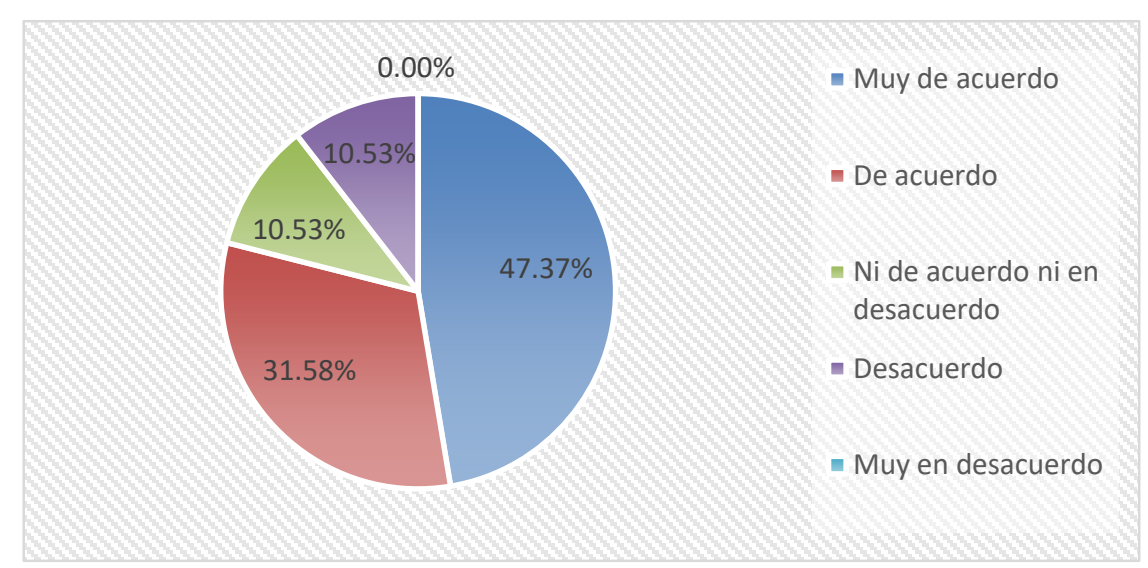

Gráfico 5. Resultados de la Pregunta 5.

Fuente: Elaboración propia.

El Gráfico 6 muestra los resultados obtenidos para la Pregunta 6: Disfruté el contenido del curso por medio de Facebook. El 47.37\% de los alumnos consideran que disfrutan el proceso de enseñanza-aprendizaje.

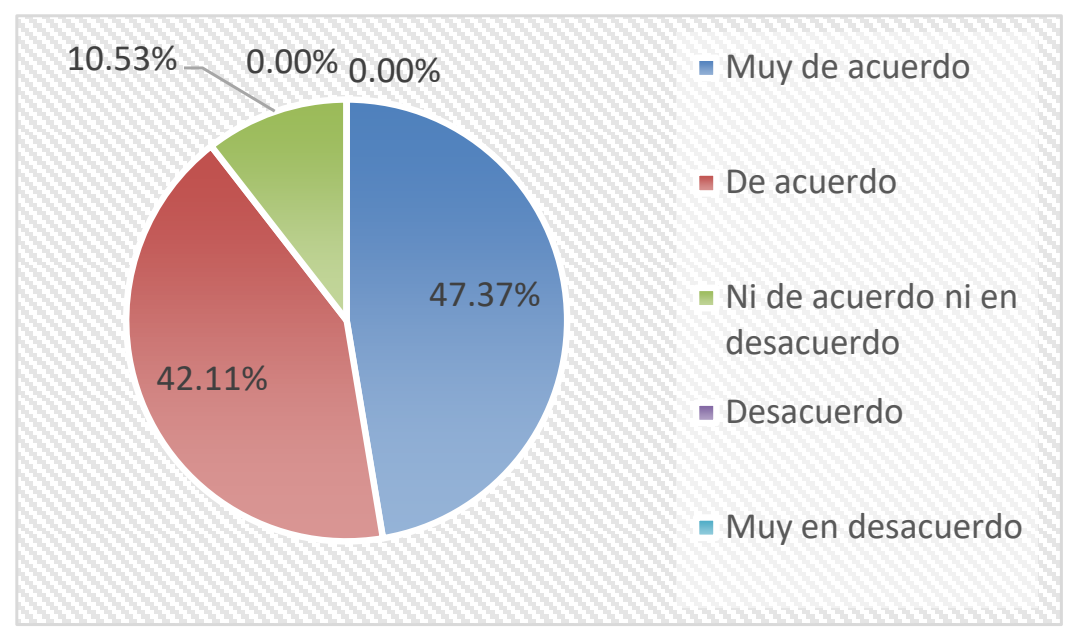

Gráfico 6. Resultados de la Pregunta 6.

Fuente: Elaboración propia.

A continuación se muestran los resultados sobre la variable "Aprendizaje" para las preguntas 7,8 y 9 del cuestionario.

El Gráfico 7 muestra los resultados obtenidos para la Pregunta 7: Mientras empleaba Facebook, estaba seguro de que aprendería. El mayor porcentaje de aceptación se ubica en la categoría Muy de acuerdo con el $42.11 \%$. 
Salas Rueda, R. I., Lugo García, J. L., Ruiz Silva, H. F. Perspectivas de los estudiantes sobre el uso de redes sociales en el proceso educativo superior

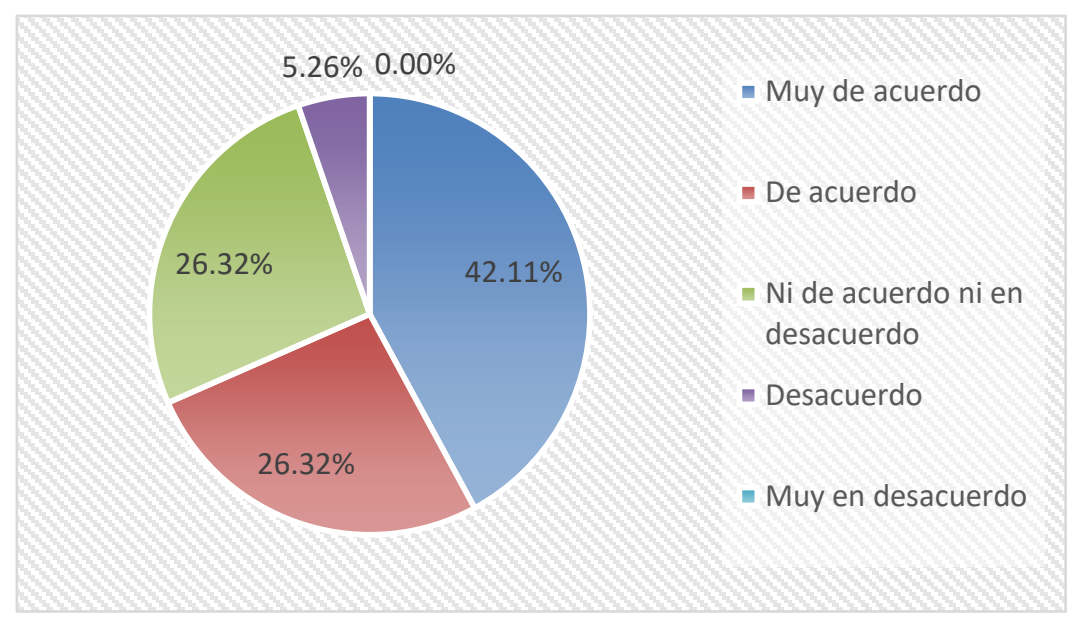

Gráfico 7. Resultados de la Pregunta 7.

Fuente: Elaboración propia.

El Gráfico 8 muestra los resultados obtenidos para la Pregunta 8: La buena organización de Facebook me permitió tener confianza de que puedo aprender. Con relación a este aspecto, las categorías Muy de acuerdo y $\mathrm{Ni}$ de acuerdo ni en desacuerdo obtienen el mayor porcentaje con el $36.84 \%$.

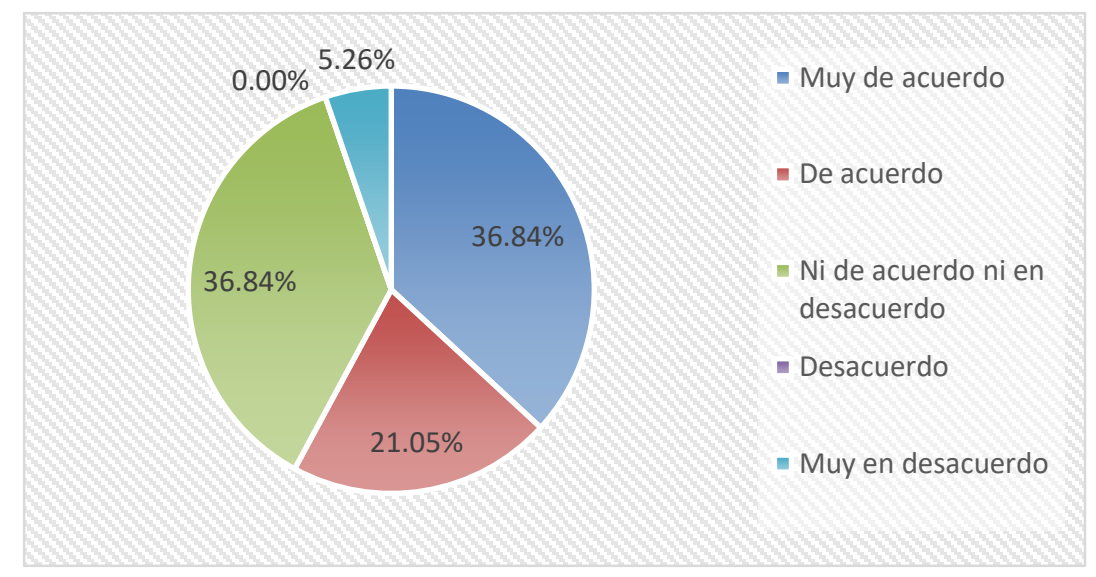

Gráfico 8. Resultados de la Pregunta 8.

Fuente: Elaboración propia.

El Gráfico 9 muestra los resultados obtenidos para la Pregunta 9: Los contenidos y la estructura de Facebook son fáciles de entender. La mayor preferencia se ubica en la categoría Muy de acuerdo con el 73.68\% 
Salas Rueda, R. I., Lugo García, J. L., Ruiz Silva, H. F. Perspectivas de los estudiantes sobre el uso de redes sociales en el proceso educativo superior

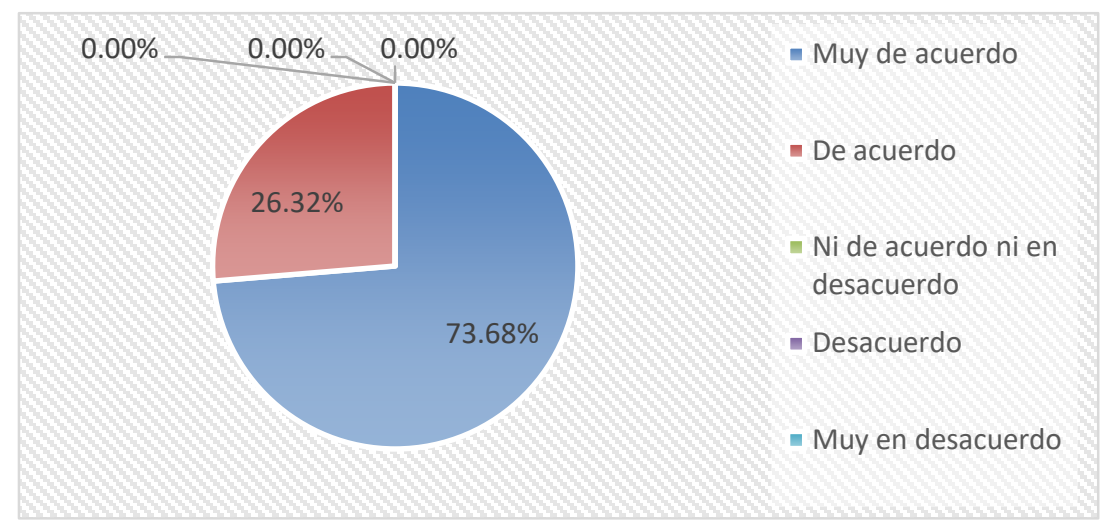

Gráfico 9. Resultados de la Pregunta 9.

Fuente: Elaboración propia.

A continuación se muestran los resultados sobre la variable "Motivación" para las preguntas 10, 11 y 12 del cuestionario.

El Gráfico 10 muestra los resultados obtenidos para la Pregunta 10: Me gustaría saber más sobre el tema discutido en Facebook. El 57.89\% de los estudiantes están en la categoría De acuerdo sobre la necesidad de conocer más sobre los contenidos.

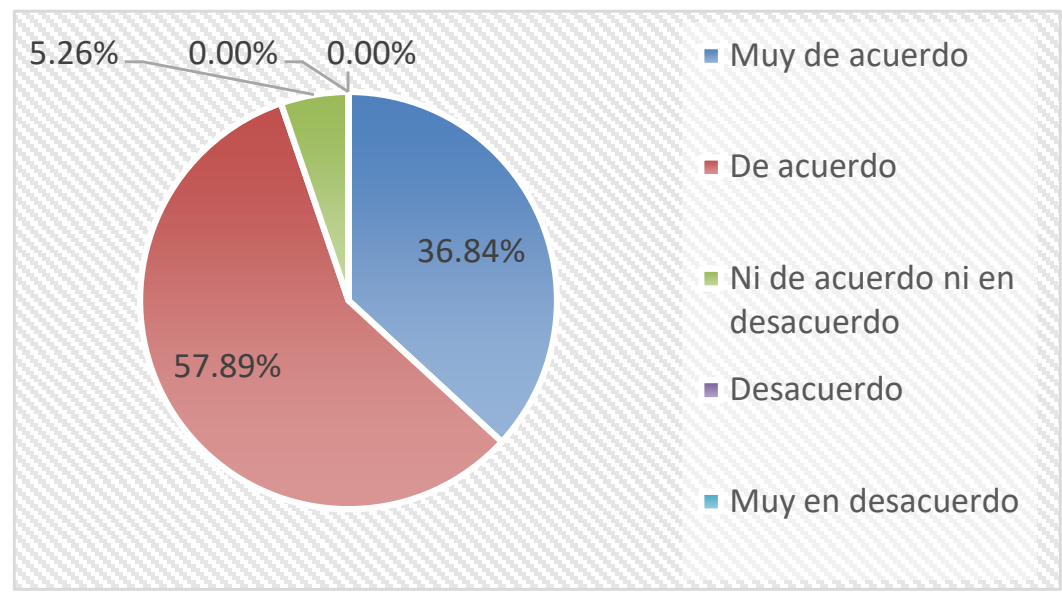

Gráfico 10. Resultados de la Pregunta 10.

Fuente: Elaboración propia.

El Gráfico 11 muestra los resultados obtenidos para la Pregunta 11: Me siento satisfecho del uso de la red social Facebook. El $47.37 \%$ de los alumnos se sienten cómodos de utilizar esta red social al ubicarse en la categoría Muy de acuerdo. Del mismo modo, el $42.11 \%$ de los usuarios se encuentran en la categoría De acuerdo. 
Salas Rueda, R. I., Lugo García, J. L., Ruiz Silva, H. F. Perspectivas de los estudiantes sobre el uso de redes sociales en el proceso educativo superior

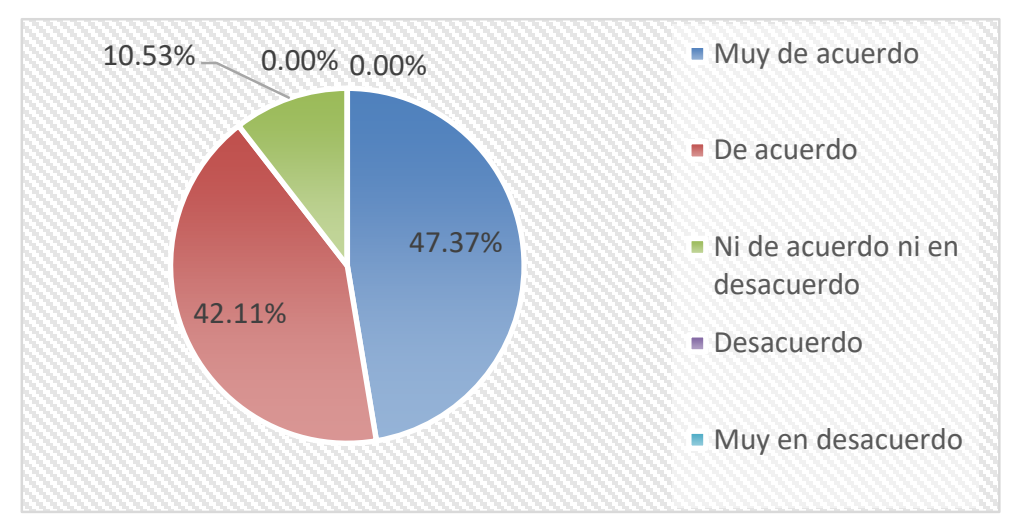

Gráfico 11. Resultados de la Pregunta 11.

Fuente: Elaboración propia.

El Gráfico 12 muestra los resultados obtenidos para la Pregunta 12: Había contenidos en Facebook que me mostraron que este material puede ser importante para otras personas. El 47.37\% de los estudiantes se encuentran en la categoría Muy de acuerdo y el $42.11 \%$ están en la categoría De acuerdo.

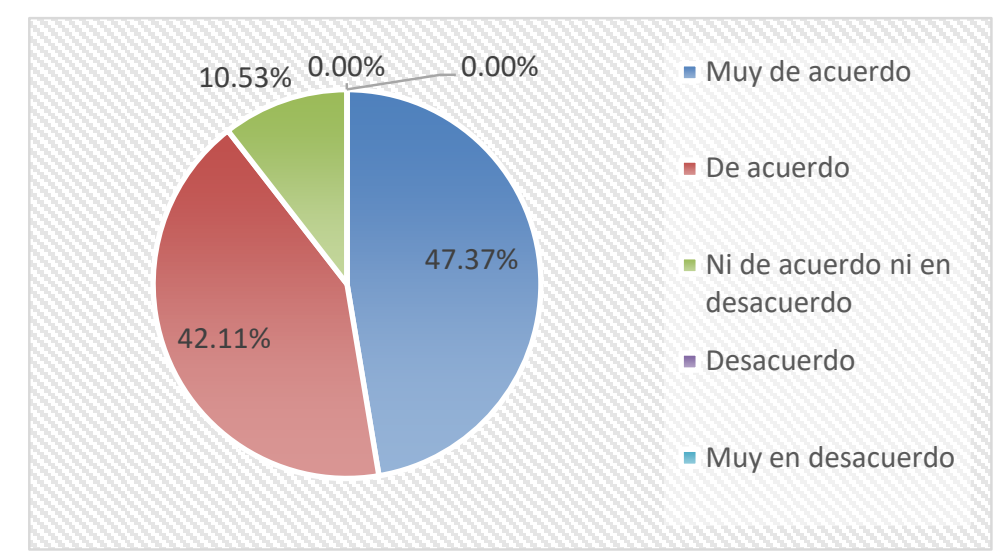

\section{Gráfico 12. Resultados de la Pregunta 12.}

Fuente: Elaboración propia.

La Tabla 2 muestra la frecuencia porcentual para las variables de Atención, Utilidad, Aprendizaje y Motivación durante el uso de la red social Facebook.

Tabla 2.

Frecuencia porcentual de las variables empleadas en este estudio.

\begin{tabular}{|c|c|c|c|c|c|}
\hline No & Respuestas & Atención & Utilidad & Aprendizaje & Motivación \\
\hline 1 & Muy de acuerdo & $29.825 \%$ & $45.614 \%$ & $50.877 \%$ & $43.860 \%$ \\
\hline 2 & De acuerdo & $49.123 \%$ & $38.596 \%$ & $24.561 \%$ & $47.368 \%$ \\
\hline 3 & Ni de acuerdo ni en desacuerdo & $14.035 \%$ & $10.526 \%$ & $21.053 \%$ & $8.772 \%$ \\
\hline 4 & Desacuerdo & $7.018 \%$ & $5.263 \%$ & $1.754 \%$ & $0.000 \%$ \\
\hline 5 & Muy en desacuerdo & $0.000 \%$ & $0.000 \%$ & $1.754 \%$ & $0.000 \%$ \\
\hline
\end{tabular}

Fuente: Elaboración propia. 
Salas Rueda, R. I., Lugo García, J. L., Ruiz Silva, H. F. Perspectivas de los estudiantes sobre el uso de redes sociales en el proceso educativo superior

\section{CONCLUSIÓN}

Los avances de la tecnología relacionados con las herramientas informáticas y aplicaciones de comunicación obligan a los responsables del proceso de enseñanzaaprendizaje a planear y organizar nuevas estrategias que permitan la asimilación del conocimiento y desarrollo de las habilidades en los estudiantes (Ellis, 2016; Paiva, Morais, Costa \& Pinheiro, 2016; Taddeo \& Barnes 2016; Vuopala, Hyvönen \& Jarvela, 2016).

El sistema de educación a distancia está compuesto por la tecnología, el aprendizaje, la enseñanza, el diseño del curso y la administración de las políticas (Moore y Kearsley, 2012). En particular, "los sistemas de redes sociales permiten una conexión humana más personal y motivadora que otras plataformas" (Rojas, 2014, p. 1).

Una de las ventajas que ofrece la red social Facebook a los usuarios de la web es la facilidad para compartir la información, las fotos, los videos y los mensajes (Llorens y Capdeferro, 2011; Pimmer, Linxen \& Gröhbiel, 2012).

En esta investigación se analiza el impacto de utilizar Facebook durante el proceso educativo superior sobre la informática considerando las variables de Atención, Utilidad, Aprendizaje y Motivación. Resulta valioso mencionar que el $42 \%$ de los estudiantes están en la categoría Muy de acuerdo respecto a que esta red social captó su atención. Del mismo modo, el $47.37 \%$ de los estudiantes opinan que les pareció que iba a ser fácil de utilizar (variable Utilidad). Por otro lado, la variable Aprendizaje sobre que los contenidos y la estructura de la red social Facebook son fáciles de entender adquiere un $73.68 \%$ de aceptación entre los usuarios. Asimismo el $47 \%$ de los alumnos están muy satisfechos de utilizar esta red social durante el proceso de enseñanza-aprendizaje (variable Motivación).

Cabe mencionar que la frecuencia porcentual más alta para la categoría Muy de acuerdo está representada por la variable Aprendizaje con el $50.87 \%$, seguida por las variables Utilidad (45.61\%), Motivación (43.86\%) y Atención (29.82\%).

Finalmente, las redes sociales representan una alternativa tecnológica del Siglo XX1 que permite mejorar las condiciones actuales del proceso educativo por medio de la colaboración y la reflexión de los participantes (Demirbilek, 2015; Lin, Hu, Hu \& Liu, 2016; Magogwe, Ntereke \& Phetlhe, 2015; Scott, 2013). 
Salas Rueda, R. I., Lugo García, J. L., Ruiz Silva, H. F. Perspectivas de los estudiantes sobre el uso de redes sociales en el proceso educativo superior

\section{REFERENCIAS}

Altinay, Z.; Saner, T.; Bahcelerli, N. M. \& Altinay, F. (2016). The Role of Social Media Tools: Accessible Tourism for Disabled Citizens. Educational Technology E Society, 19(1), 89-99.

Demirbilek, M. (2015). Social media and peer feedback: What do students really think about using Wiki and Facebook as platforms for peer feedback? Active Learning in Higher Education, 16, 211-224. doi:10.1177/1469787415589530

Ellis, A. (2016). Qualitatively different university student experiences of inquiry: Associations among approaches to inquiry, technologies and perceptions of the learning environment. Active Learning in Higher Education, 17, 13-23, doi:10.1177/1469787415616721

Ersoy, M. \& Güneyli, A. (2016). Social Networking as a Tool for Lifelong Learning with Orthopedically Impaired Learners. Educational Technology E Society, 19(1), 4152.

Gallardo, E.; Marqués, L. \& Bullen, M. (2015). El estudiante en la educación superior: Usos académicos y sociales de la tecnología digital. Universities and Knowledge Society Journal, 12(1). 25-37. doi:dx.doi.org/10.7238/rusc.v12i1.2078

García Aretio, L. (1999). Historia de la educación a distancia. Revista Iberoamericana de Educación a Distancia, 2(1), 8-27.

González Ortiz, J. J.; Tornel Abellán, M. \& Jiménez Hernández, D. (2014). La enseñanza virtual y los métodos activos en educación superior, en J. F. Durán Medina, \& I. Durán Valero, (Eds.), La era de las TIC en la nueva docencia (pp. 199210). Madrid: McGraw Hill Education.

Huang, T. Y.; Wu, H. L.; She, H. C. \& Lin, Y. R. (2014). Enhancing Students' NOS Views and Science Knowledge Using Facebook-based Scientific News. Educational Technology \& Society, 17 (4), 289-301.

Huang, J. J. S.; Yang, S. J. H.; Huang, Y. M. \& Hsiao, I. Y. T. (2010). Social Learning Networks: Build Mobile Learning Networks Based on Collaborative Services. Educational Technology \& Society, 13 (3), 78-92.

Lin, X.; Hu, X.; Hu, Q. \& Liu, Z. (2016). A social network analysis of teaching and research collaboration in a teachers' virtual learning community. British Journal of Educational Technology, 47, 302-319. doi:10.1111/bjet.12234

Llorens, F. \& Capdeferro, N. (2011). Posibilidades de la plataforma Facebook para el aprendizaje colaborativo en línea. Revista de Universidad y Sociedad del Conocimiento, $8(2), 31-45$. 
Salas Rueda, R. I., Lugo García, J. L., Ruiz Silva, H. F. Perspectivas de los estudiantes sobre el uso de redes sociales en el proceso educativo superior

Magogwe, J. M.; Ntereke, B. \& Phetlhe, K. R. (2015). Facebook and classroom group work: A trial study involving University of Botswana Advanced Oral Presentation students. British Journal of Educational Technology, 46, 1312-1323. doi: 10.1111/bjet.12204

Martínez Rivera, O. (2014). Modelos de enseñanza y aprendizaje en la Universidad: Redes sociales y aplicaciones móviles para la generación de trabajos en grupo, en J. F. Durán Medina, \& I. Durán Valero, (Eds.), La era de las TIC en la nueva docencia (pp. 267-290). Madrid: McGraw Hill Education.

Moore, M. \& Kearsley, G. (2012). Distance education: A systems view of online learning. California: Cengage Learning.

Olarte Larrea, J. J.; Domínguez Pérez, C.; Elizondo, A. J. \& García Izquierdo, F. J. (2014). Incorporado Facebook como herramienta de apoyo en los trabajos de fin de grado de ingeniería informática, en J. F. Durán Medina, \& I. Durán Valero, (Eds.), La era de las TIC en la nueva docencia (pp. 351-362). Madrid: McGraw Hill Education.

Paiva, J.; Morais, C.; Costa, L. \& Pinheiro, A. (2016). The shift from e-learning to learning: Invisible technology and the dropping of the " $\mathrm{e}$ ". British Journal of Educational Technology, 47, 226-238. doi: 10.1111/bjet.12242

Pimmer, C.; Linxen, S. \& Gröhbiel, U. (2012). Facebook as a learning tool? A case study on the appropriation of social network sites from mobile phones in developing countries. British Journal of Educational Technology, 43, 726-738. doi: 10.1111/j.1467-8535.2012.01351.x

Reigeluth, C. M. (2012). Instructional theory and technology for the new paradigm of education. Revista de Educación a Distancia, 32, 1-18.

Rojas Kramer, C. A. (2014). Uso de Facebook en ámbitos educativos universitarios: consideraciones y recomendaciones. Revista Apertura, 6 (2), 1-12.

Scott, K. M. (2013). Does a university teacher need to change e-learning beliefs and practices when using a social networking site? A longitudinal case study. British Journal of Educational Technology, 44, 571-580. doi:10.1111/bjet.12072

Simonson, M.; Smaldino, S.; Albright, M. \& Zvacek, S. (2012). Teaching and Learning at a Distance. Foundations of Distance Education. Nueva York: Pearson.

Taddeo, C. \& Barnes, A. (2016). The school website: Facilitating communication engagement and learning. British Journal of Educational Technology, 47, 421-436. doi:10.1111/bjet.12229 
Salas Rueda, R. I., Lugo García, J. L., Ruiz Silva, H. F. Perspectivas de los estudiantes sobre el uso de redes sociales en el proceso educativo superior

Valerio, G.; Herrera Murillo, D.; Villanueva, F.; Herrera Murillo, N. \& Rodríguez Martínez, M. C. (2015). Relación entre los formatos de publicación y el engagement digital: estudio de las páginas de Facebook de las universidades mexicanas. Universities and Knowledge Society Journal, 12(1), 50-54. doi:dx.doi.org/10.7238/rusc.v12i1.1887

Vuopala, E; Hyvönen, P. \& Jarvela, S. (2016). Interaction forms in successful collaborative learning in virtual learning environments. Active Learning in Higher Education, 17, 25-38. doi:10.1177/1469787415616730

\section{AUTORES:}

\section{Ricardo Adán Salas Rueda}

Doctor en Diseño de Nuevas Tecnologías, egresado de la Universidad Autónoma Metropolitana (UAM). Durante 13 años ha impartido diversos cursos en el nivel de licenciatura y posgrado. Candidato a investigador nacional por parte del Sistema Nacional de Investigadores (SNI). Responsable del Grupo de Investigación "Sistemas usables" en la Universidad La Salle.

http://orcid.org/0000-0002-6689-4460

\section{José Luis Lugo García}

Maestro de educación secundaria en Mecánica Automotriz. Ingeniero Civil. Maestría en Planeación. Maestría en Finanzas. Durante 16 años impartió clases en secundaria. Fue director durante 8 años en secundaria y preparatoria (particular). Laboró en la Universidad ISEC durante 23 años a nivel Preparatoria, licenciatura y maestría y en la Universidad la Salle durante 21 años a nivel licenciatura.

https://www.researchgate.net/researcher/2107256668_Jose_Luis_Lugo_Garcia

\section{Héctor Fernando Ruiz Silva}

Actualmente, es estudiante de la carrera de Gestión de Negocios y Tecnologías de la información por la Universidad La Salle. Cursa el 6to semestre de su carrera mientras labora en una institución financiera en el área de desarrollo de aplicaciones enfocándose en el uso e implementación de herramientas ETL atendiendo requerimientos específicos de los diferentes usuarios internos. Tiene un amplio interés en el área de Big Data, análisis de datos e inteligencia de negocios, por lo cual, estuvo de acuerdo en participar en la elaboración de este estudio con el fin de obtener experiencia en este campo y desarrollar sus conocimientos en sus áreas de interés. 